\title{
ORTODONTIA INTERCEPTATIVA NO TRATAMENTO DE MORDIDA CRUZADA POSTERIOR BILATERAL E MORDIDA ABERTA ANTERIOR: RELATO DE CASO
}

\author{
INTERCEPTIVE ORTHODONTICS IN THE TREATMENT OF BILATERAL \\ POSTERIOR CROSSBITE AND ANTERIOR OPEN BITE: CASE REPORT
}

\author{
Alessandra Rodrigues Fonseca TAVARES'; Cyntia R. A. ESTRELA²; Priscilla Cardoso LAZARI-CARVALHO 3 \\ 1 - Especialista em Ortodontia e aluna do Programa de Pós-Graduação em Odontologia da Faculdade de Odontologia do Centro Universitário de \\ Anápolis - UniEVANGÉLICA. \\ 2 - Doutora em Biologia celular e molecular, professora do curso de Odontologia e Programa de Pós-Graduação da Faculdade de Odontologia do \\ Centro Universitário de Anápolis - UniEVANGÉLICA. \\ 3 - Doutora em Clínica Odontológica-Prótese Dental, Professora do curso de Odontologia e Programa de Pós-Graduação da Faculdade de \\ Odontologia do Centro Universitário de Anápolis - UniEVANGÉLICA.
}

\section{RESUMO}

O diagnóstico e a intervenção precoce da má oclusão possibilitam um crescimento adequado do complexo maxilo-mandibular. O objetivo deste trabalho é apresentar um relato de caso clínico de um paciente em fase de dentadura mista que apresentava mordida aberta anterior e mordida cruzada posterior bilateral. Foi proposto a instalação de um disjuntor maxilar tipo Haas. Considerações finais: Após a instalação do aparelho o paciente interrompeu o hábito de sucção digital que possuía. A expansão maxilar promovida pelo expansor Haas associada à interrupção do hábito resultaram na correção da mordida aberta e mordida cruzada posterior bilateral. Paciente faz uso de placa de Hawley para contenção há 9 meses.

Palavras-chave: Atresia maxilar; Expansão rápida da maxila; Má oclusão; Mordida aberta.

\section{INTRODUÇÃO}

A má oclusão é caracterizada pela desordem de desenvolvimento que afeta o complexo craniofacial, sendo resultado da interação de fatores genéticos e ambientais, que comprometem aspectos funcionais e estéticos dos indivíduos ${ }^{1,2}$. Maus hábitos e respiração bucal podem desempenhar um papel na etiopatogenia da má oclusão. Sempre que estes problemas são encontrados associados à má oclusão, devem ser eliminados a fim de garantir um meio funcional adequado para o crescimento fisiológico ${ }^{3}$. Devido a sua alta prevalência e por apresentar impacto negativo na vida do indivíduo, a má oclusão é considerada um problema de saúde pública $^{3,4}$. Tratar o paciente antes que este atinja a dentição permanente pode reduzir o número de intervenções posteriores e acelerar um tratamento futuro. A ortodontia preventiva e interceptativa tem como objetivo prevenir ou atenuar problemas oclusais que estejam ocorrendo no período de transição da dentição decídua para a permanente ${ }^{5}$. A prevenção e interceptação ortodôntica podem minimizar problemas de desenvolvimento. $\mathrm{O}$ tratamento interceptativo envolve a remoção de interferências existentes, eliminando a necessidade de tratamento ortodôntico na dentição permanente ou diminuindo a severidade do desenvolvimento da má oclusão.

O objetivo do presente caso clínico foi relatar a importância da aplicação da ortodontia interceptativa em uma criança de 9 anos e dentição mista com mordida cruzada posterior e mordida aberta anterior, apresentando alternativa de tratamento que permitiu a resolução do caso.

\section{RELATO DE CASO}

Paciente A. A. do gênero masculino, leucoderma, com 9 anos e 2 meses de idade, procurou atendimento odontológico acompanhado de seu responsável. A queixa principal do paciente e seu responsável foi a estética comprometida (Figura 1).

Na anamnese, o responsável relata o hábito de sucção digital. No exame clínico intraoral, observou-se dentição mista com presença dos incisivos e primeiros pré-molares e molares permanentes, observou-se também atresia do arco dental superior e presença de mordida cruzada posterior bilateral e mordida aberta anterior (Figura 2).

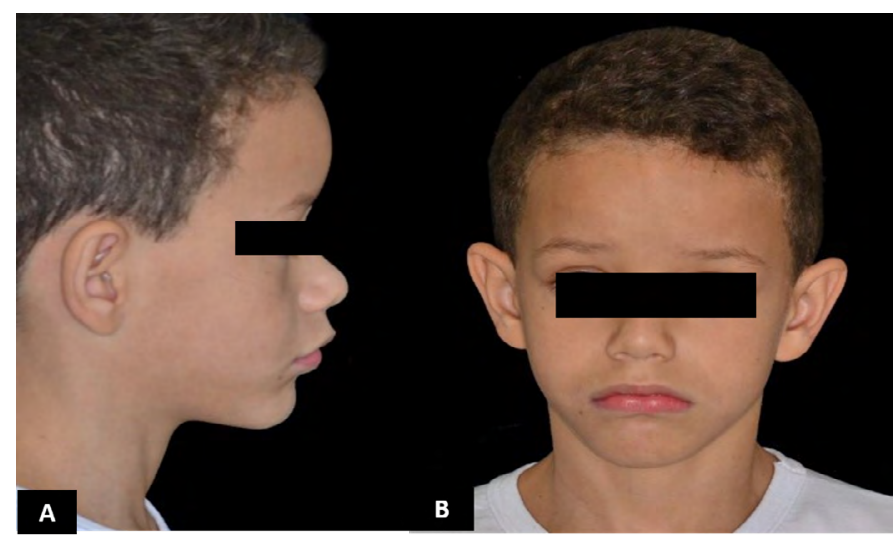

Figura 1 - (A) Foto inicial extrabucal de perfil; (B) Foto inicial extrabucal frontal. 
Foi solicitada a documentação ortodôntica como exame complementar (Figura 3). O Plano de tratamento proposto foi confecção e instalação de um expansor de maxila do tipo HAAS.

Para a confecção foi realizada moldagem dos arcos com alginato e confecção dos modelos com gesso do tipo III. Os modelos foram enviados ao laboratório especializado para a confecção do aparelho expansor.

O expansor foi instalado através da cimentação das bandas nos primeiros molares superiores com ionômero de vidro (VitroCem, DFL), foi utilizada resina composta fotopolimerizável (Natural Shade, DFL) para estabilizar a alça nos caninos superiores (Figura 4).

Após a instalação, foi orientado ao responsável que fizesse a ativação com a chave ativadora realizando $2 / 4$ de volta duas vezes ao dia, uma pela manhã e outra no período noturno, totalizando 1 volta na chave ativadora por dia. A ativação foi feita por um período de 14 dias, com visitas semanais ao dentista para supervisionar a expansão. O responsável foi informado que seria normal a criança sentir desconforto nos primeiros dias, podendo ocorrer a abertura de um diastema entre os incisivos superiores, mas que logo seria corrigido naturalmente. Foi orientada uma dieta mais pastosa nos primeiros dias e que se evitasse alimentos

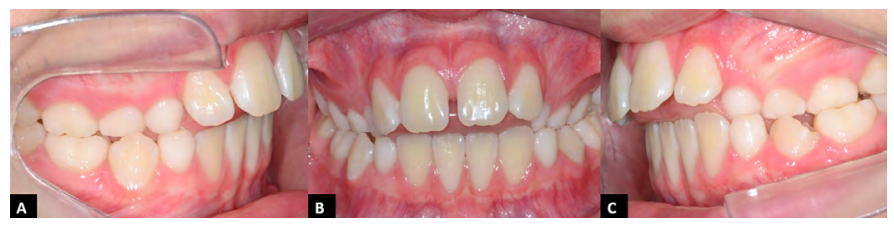

Figura 2 - (A) Foto inicial intrabucal lateral direita; (B) Foto inicial frontal; (C) Foto inicial lateral esquerda.

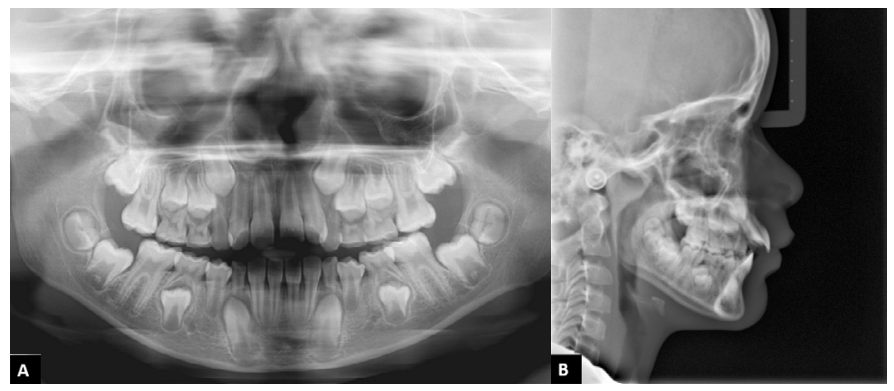

Figura 3 - (A) Radiografia panorâmica inicial; (B) Telerradiografia perfil inicial.

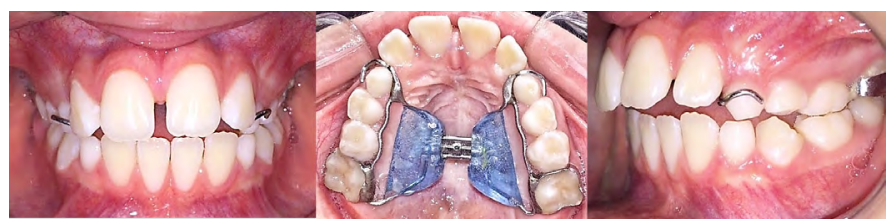

Figura 4 - (A) Foto após a instalação do expansor HAAS lateral direita; (B) Foto oclusal; (C) Foto lateral esquerda.

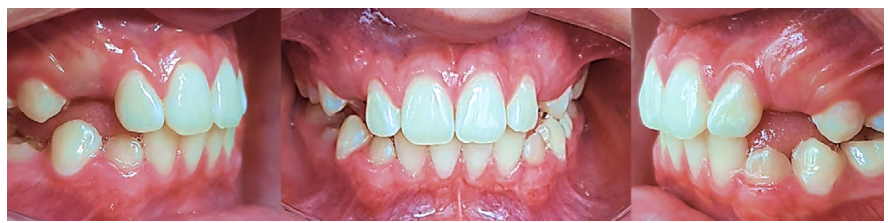

Figura 8 - (A) Foto lateral direita após 6 meses da remoção do HAAS; (B) Foto frontal; (C) Foto lateral esquerda. pegajosos e/ou duros com intuito de preservar a qualidade do aparelho expansor.

Após 14 dias observou-se a sobrecorreção da mordida pelo toque da cúspide palatina do primeiro molar superior na cuspide vestibular do primeiro molar inferior e abertura de diastema entre os incisivos superiores, interrompendo então a ativação do expansor. Após a interrupção da ativação o aparelho foi mantido em posição por 120 dias para que ocorresse a neoformação óssea da sutura palatina.

Transcorrido esse período, foi observado o fechamento do diastema entre os incisivos, correção da mordida aberta anterior e da mordida cruzada posterior bilateral (Figura 5), sendo assim o aparelho foi removido e realizada uma nova moldagem com alginato e obtenção de novos modelos para que fosse feita uma Placa de Hawley para contenção (Figura 6). Uma nova telerradiografia foi solicitada (Figura 7) Foi orientado ao paciente e seu responsável que fizesse a utilização da contenção diariamente, removendo somente durante as refeições, pelo período de 6 meses.

O controle do caso tem sido realizado com consultas periódicas ao consultório odontológico, observando a estabilidade dos resultados 6 e 9 meses após a remoção do expansor (Figuras 8 e 9).

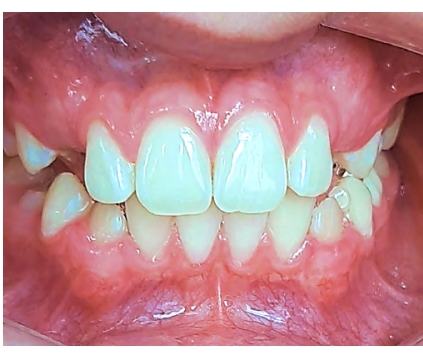

Figura 5 - Foto logo após a remoção do HAAS.

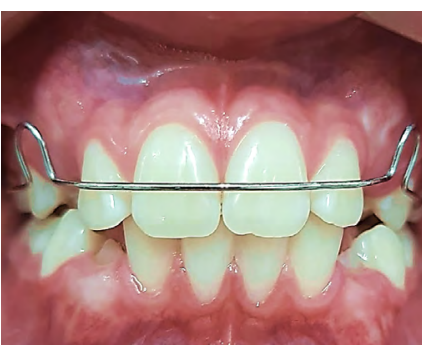

Figura 6 - Foto com a contenção.

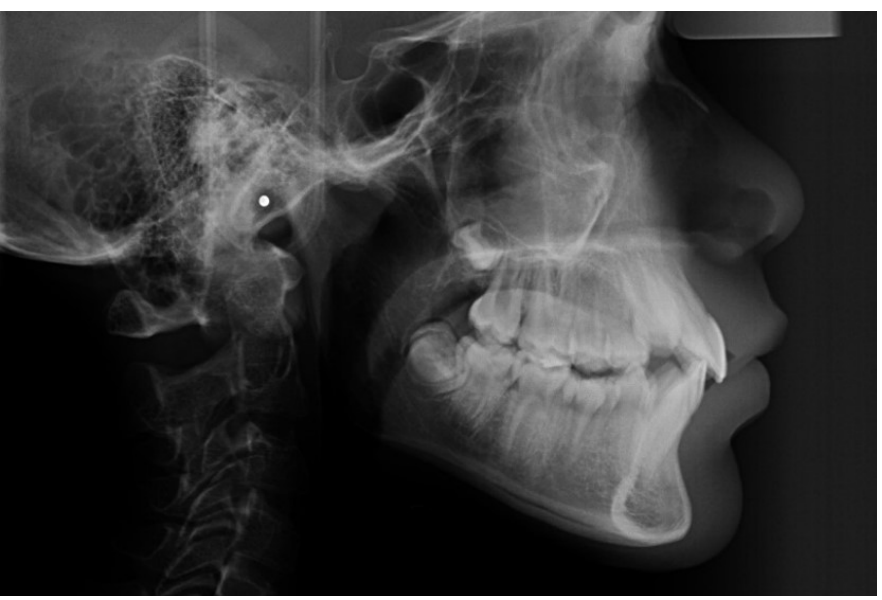

Figura 7 - Telerradiografia perfil após a remoção do HAAS.

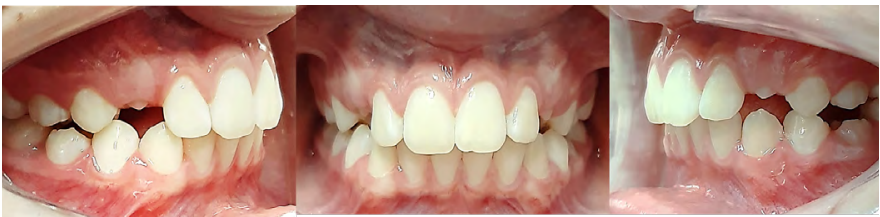

Figura 9 - (A) Foto lateral direita após 9 meses da remoçãp do HAAS; (B) Foto lateral esquerda; (C) Foto lateral direita. 


\section{DISCUSSÃO}

Desordens miofuncionais orofaciais incluem disfunção dos lábios, língua e ou orofaringe que interferem no crescimento normal, ou no desenvolvimento e função de outras estruturas orais ${ }^{6}$. Hábitos orais incluem sucção digital, sucção dos lábios, roer unhas, respiração bucal, interposição lingual, dentre outros. Seus efeitos dependem do início e da duração do hábito. Hábitos de sucção podem resultar em alterações a longo prazo e afetar o sistema estomatognático, levando a um desequilíbrio entre a musculatura externa e interna, contribuindo também para alterações no desenvolvimento ósseo ${ }^{7}$. Estudos têm demostrado uma significante associação entre hábito de sucção digital e presença de mordida aberta anterior e mordida cruzada posterior. A sucção pode causar a protrusão dos incisivos superiores e pré-maxila, deglutição atípica, mordida aberta anterior e mordida cruzada posterior. A mordida cruzada posterior ocorre devido à posição baixa da língua durante a sucção, com falta de estímulo no palato e um aumento da atividade dos músculos das bochechas causando então uma alteração da pressão da musculatura na arcada superior ${ }^{3}$. A expansão rápida da maxila é um procedimento ortopédico efetivo que tem sido utilizado rotineiramente em pacientes em crescimento há mais de meio século. O objetivo da expansão rápida da maxila é abrir a sutura palatina proporcionando largura maxilar adequada ${ }^{8,9}$. Neste caso com a instalação do aparelho expansor, o paciente interrompeu o hábito de sucção, e então a mordida aberta foi corrigida. A disjunção promovida pelo aparelho HAAS resultou na correção da mordida cruzada bilateral. Pouco se sabe sobre o real benefício da ortodontia interceptativa ${ }^{10}$. Quando a ortodontia interceptativa é analisada como procedimento único e conceituada apenas como tratamento realizado antes dos 11 anos de idade, os resultados de estudos demonstram que esse tipo de abordagem não gera benefício adicional em relação ao tratamento realizado mais tarde. No entanto, quando as modalidades são avaliadas isoladamente, os resultados são diferentes ${ }^{5}$. O tratamento da mordida aberta anterior gera mudanças dentoalveolares significativas na região anterior, corrigindo-a por meio de extrusão e verticalização dos incisivos ${ }^{11}$. Nas mordidas cruzadas posteriores, os resultados são mantidos, conforme acompanhamento por até três anos, pós-expansão, segundo estudos ${ }^{5}$. Fica claro como uma intervenção interceptativa da má oclusão é benéfica para evitar o desequilíbrio no desenvolvimento esquelético facial em pacientes em crescimento ${ }^{12}$. A prevenção é uma alternativa eficiente, que pode simplificar ou até mesmo evitar tratamentos corretivos ortodônticos futuros.

\section{CONCLUSÃO}

Na literatura vemos a contestação da eficácia da ortodontia preventiva e interceptativa. No entanto o impacto negativo da má oclusão na dentição mista em relação à qualidade de vida relacionada à saúde bucal da criança é comprovado. O diagnóstico precoce torna-se importante na prevenção de más oclusões da dentadura permanente, pois a presença destas alterações na dentadura decídua pode ser preditiva. No presente estudo o uso do HAAS promoveu a expansão da maxila, impediu o hábito de sucção digital, resultando na correção da mordida cruzada posterior bilateral e da mordida aberta anterior, além da melhora da estética do paciente contribuindo para uma melhor qualidade de vida. $\mathrm{O}$ paciente está sendo acompanhado e observa-se o desenvolvimento de uma oclusão equilibrada pela intervenção proposta.

\section{REFERÊNCIAS}

01. Zou J, Meng M, Law CS, Rao Y, Zhou X. Common dental diseases in children and malocclusion. Int J Oral Sci. 2018;10(1):1-7.

02. Moreira AF, Pinto LS, Pinto KVA, Côrreia PG, Jeziorski SAZ, Velasque $\mathrm{KS}$, et al. Impacto da má oclusão na dentição decídua e permanente na qualidade de vida de crianças e adolescentes: revisão de literatura. Rev Bras Odontol. 2015;72(1/2):70.

03. Grippaudo C, Paolantonio EG, Antonini G, Saulle R, La Torre G, Deli R. Associazione fra abitudini viziate, respirazione orale e malocclusione. Acta Otorhinolaryngol Ital. 2016;36(5):386-94.

04. Bauman JM, Souza JGS, Bauman CD, Flório FM. Epidemiological pattern of malocclusion in brazilian preschoolers. Ciência e Saúde Coletiva. 2018;23(11):3861-8.

05. Artese F. Olhando a Ortodontia Interceptativa de uma forma mais abrangente: o que realmente podemos oferecer? Dent Press J Orthod. 2019;24(5):7-8.

06. D'Onofrio L. Oral dysfunction as a cause of malocclusion. Orthod Craniofacial Res. 2019;22(S1):43-8.

07. Matsumoto MAN, Romano FL, Ferreira JTL, Valério RA. Open bite: Diagnosis, treatment and stability. Braz Dent J. 2012;23(6):768-78.

08. McNamara JA, Lione R, Franchi L, Angelieri F, Cevidanes LHS, Darendeliler MA, et al. The role of rapid maxillary expansion in the promotion of oral and general health. Prog Orthod [Internet]. 2015;16(1):1-7.

09. OG Silva F, L Capelozza F. Expansão Rápida da Maxila: Considerações Gerais e. Ortodontia. 1988;21(1):61-81.

10. Sunnak R, Johal A, Fleming PS. Is orthodontics prior to 11 years of age evidence-based? A systematic review and meta-analysis. J Dent. 2015;43(5):477-86.

11. Pisani L, Bonaccorso L, Fastuca R, Spena R, Lombardo L, Caprioglio A. Systematic review for orthodontic and orthopedic treatments for anterior open bite in the mixed dentition. Prog Orthod. 2016;17(1):28.

12. Kragt L, Dhamo B, Wolvius EB, Ongkosuwito EM. The impact of malocclusions on oral health-related quality of life in children-a systematic review and meta-analysis. Clin Oral Investig. 2016;20(8):1881-94.

\section{ABSTRACT}

Diagnosis and early intervention of malocclusion enable adequate growth of the mandibular maxillary complex. The aim of this paper is to report a case of a patient with mixed dentition who presented anterior open bite and bilateral posterior crossbite. It has been proposed to install a Haas type jaw breaker. Final considerations: After the device was installed, the patient discontinued his digital sucking habit. The maxillary expansion promoted by Haas expander associated with the interruption of the habit resulted in the correction of the open bite and bilateral posterior crossbite. Patient has been using Hawley plaque for retention for 9 months.

Keywords: Maxillary atresia; Rapid maxillary expansion; Malocclusion; Open bite. 


\section{AUTOR PARA CORRESPONDÊNCIA}

Alessandra Rodrigues Fonseca Tavares

Faculdade de Odontologia do Centro Universitário de

Anápolis - UniEVANGÉLICA

Av. Universitária Km 3,5 - Cidade Universitária - Anápolis-

GO, CEP: 75083-55.

E-mail: alessaftavares@gmail.com 